\title{
The Efficacy of Transdermal Rivastigmine in Mild to Moderate Alzheimer's Disease with Concomitant Small Vessel Cerebrovascular Disease: Findings from an Open-Label Study
}

This article was published in the following Dove Press journal:

Clinical Interventions in Aging

\author{
Chathuri Yatawara' \\ Fatin Zahra Zailan' \\ Esther Vanessa Chua' \\ Linda Lay Hoon Lim' \\ Eveline Silva' \\ Joanna Sihan Wang' \\ Adeline $\mathrm{Ng}^{\prime}$ \\ Kok Pin $\mathrm{Ng}^{\prime}$ \\ Nagaendran Kandiah ${ }^{1-3}$ \\ 'Department of Neurology, National \\ Neuroscience Institute, Singapore, \\ Singapore; ${ }^{2}$ Duke-NUS Medical School, \\ Singapore, Singapore; ${ }^{3}$ Lee Kong Chian \\ School of Medicine-Imperial College \\ London, Nanyang Technological \\ University, Singapore, Singapore
}

Background: Rivastigmine is used to treat cognitive impairment in Alzheimer's disease (AD); however, the efficacy of Rivastigmine in patients with AD and concomitant small vessel cerebrovascular disease (svCVD) remains unclear. We investigated the effectiveness of Rivastigmine Patch in patients with $\mathrm{AD}$ and svCVD.

Methods: In this open-label study, 100 patients with $\mathrm{AD}$ and MRI confirmed svCVD received $9.5 \mathrm{mg} / 24$ hours Rivastigmine transdermal treatment for 24 weeks. The primary outcome was global cognition indexed using the ADAS-Cog. Secondary outcomes included clinical-rated impression of change (indexed using (ADCS-CGIC), activities of daily living (indexed using ADCS-ADL) and side effects.

Results: Overall, performance on the ADAS-Cog after 24 weeks deteriorated by 1.78 (SD = 5.29 ) points. Fifty-two percent of the sample demonstrated improvement or remained stable, while $48 \%$ demonstrated worsening of ADAS-Cog scores. Of the $52 \%$, significant improvement ( 2 or more-point decline) on the ADAS-Cog was observed in $25 \%$ of the sample, with a mean change of -5.08 ( $\mathrm{SD}=3.11)$. A decline on the ADAS-Cog was observed in $48 \%$ of the sample, with a mean change of $6(\mathrm{SD}=2.98)$ points. Cognitive outcome did not interact with severity of SVCVD. ADCS-ADL scores remained stable from baseline to week 24 and ADCS-CGIC reports indicated that $81 \%$ of the patients remained stable after treatment. Side effects were reported by $16 \%$ of the patients, with contact dermatitis being the most common.

Conclusion: Our findings suggest that Rivastigmine may have a role in the management of patients having $\mathrm{AD}$ and concomitant mild-severe svCVD, with minimal side effects.

Keywords: rivastigmine, Alzheimer's disease, small vessel cerebrovascular disease, treatment

\section{Introduction}

Alzheimer's disease (AD) and cerebrovascular disease are leading causes of dementia, with cerebrovascular disease, specifically small vessel cerebrovascular disease (svCVD) co-existing in one-third of AD patients worldwide. ${ }^{1}$ In Asia, the comorbidity of $\mathrm{AD}$ and svCVD is believed to be even higher at $50 \%{ }^{2}$ Cholinesterase inhibitors such as rivastigmine is an approved treatment for the symptomatic management of cognitive deficits in AD. This inhibitor reduces the activity of the esterase enzyme and results in improved activity of the acetylcholine neurotransmitter, which has been clearly
Correspondence: Nagaendran Kandiah National Neuroscience Institute, Level 3 Clinical Staff Office, II Jalan Tan Tock

Seng, Singapore, 308433, Singapore

Tel +656357 7171

Fax +6563577137

Email Nagaendran.Kandiah@singhealth. com.sg 
established to reduce cognitive decline in patients with $\mathrm{AD}^{3-5}$ There is evidence that levels of acetylcholine are also reduced in patients with cerebrovascular disease, ${ }^{6}$ and emerging evidence suggests that Rivastigmine may have stronger treatment effects in patients with comorbid $\mathrm{AD}$ and $\mathrm{svCVD}^{7}$ and less side effects ${ }^{8}$ compared to $\mathrm{AD}$ patients without svCVD. Collectively these studies suggest that patients with $\mathrm{AD}$ having concomitant svCVD may experience greater clinical benefits with Rivastigmine treatment than AD patients without sVCVD; however, direct evidence for the role of rivastigmine in patients with $\mathrm{AD}$ and concomitant SVCVD is limited.

The presence of comorbid SVCVD is most reliably detected on MRI FLAIR images in the form of white matter hyperintensities (WMH). WMH have been associated with a twofold increased risk of dementia, a threefold increased risk of stroke and a threefold increased risk of death. ${ }^{9}$ To date, MRI confirmed svCVD patients have yet to be assessed for their response to Rivastigmine. Due to the high prevalence of svCVD among Asian patients with $\mathrm{AD}$, there is an urgent need to study the effectiveness of Rivastigmine patch in subjects with svCVD. In an openlabel trial, we sought to identify the effectiveness of Rivastigmine Patch in patients with AD having MRI confirmed svCVD. We hypothesized that over a 24-week period, patients with $\mathrm{AD}$ having concomitant SvCVD treated with Rivastigmine patch $9.5 \mathrm{mg} / 24$ hours will experience improvements in cognition and daily function.

\section{Methods}

\section{Participants}

One hundred consecutive patients diagnosed with $\mathrm{AD}$ and MRI confirmed svCVD were recruited from a memory clinic at National Neuroscience Institute, Singapore. Mild to moderate probable $\mathrm{AD}$ was diagnosed by cognitive neurologists using the NIA-AA Criteria ${ }^{10}$ and a Clinical Dementia Rating $(\mathrm{CDR})^{11}$ scale of 1 or 2 . The diagnosis of sVCVD was based on the presence of moderate to severe white matter disease, indexed using the Fazekas scale score $^{12}$ of $2-3$.

Inclusion criteria were as follows: 1) age between 50 and 85 years; 1) MRI brain (with T2 or FLAIR sequences) performed within a 12-month period from time of recruitment; and 3) a Mini-Mental State Examination (MMSE) ${ }^{13}$ score of 12-28. Exclusion criteria were as follows: 1) severe neurological, psychiatric or systemic disease which in the opinion of the clinician could interfere with trial assessments;
2) use of any investigational drugs, antipsychotic or dopaminergic agents, NMDA receptor antagonist, cholinesterase inhibitors or anti-cholinergic agents during the last 4 weeks prior to recruitment; and 3) known skin allergy or previous allergic reaction to transdermal treatment.

\section{Study Design}

In this open-label study, each patient received transdermal Rivastigmine treatment for 24 weeks. For the first 4 weeks of the study, subjects received Rivastigmine patch $4.6 \mathrm{mg} /$ 24 hours. Thereafter, subjects received Rivastigmine patch $9.5 \mathrm{mg} / 24$ hours. Dose adjustments (interruptions or downtitrations for up to 2 weeks at a time, for a maximum of 2 times) were permitted to address perceived safety or tolerability issues. The Rivastigmine patch was applied by the patient or their caregivers to clean, dry, hairless skin and worn for 24 hours, during which normal activities including bathing were allowed. Cognitive assessments were conducted at baseline, week 16 and week 24 .

This study was conducted in accordance with the ethical principles that have their origins in the Declaration of Helsinki and local clinical research guidelines. The study received approval from the SingHealth Centralized Review Board and was registered with the Health Science Authority Singapore, CTA9900218 (registered $28 / 11 / 2014$ ). Prior to data collection, written informed consent was received from all patients.

\section{Outcomes}

The primary outcome was change in global cognition, measured using the ADAS-Cog. ${ }^{14}$ The ADAS-Cog measures 11 cognitive functions: spoken language ability, comprehension of spoken language, recall of test instructions, wordfinding difficulty, following commands, naming objects, construction drawing, ideational praxis, orientation, word recall and word recognition. The total score ranges from 0 to 70 , with a higher score indicating greater impairment.

A secondary outcome included clinician's impression of global change, rated using the Alzheimer's Disease Cooperative Study - Clinical Global Impression of Change scale (ADCS-CGIC). ${ }^{15}$ The ADCS-CGIC reports change from baseline using a seven-point scale, where 1 represents marked improvement and 7 represents marked worsening. A second secondary outcome was change in daily functioning, measured using the Alzheimer's Disease Cooperative Study - Activities of Daily Living (ADCSADL) scale. ${ }^{16}$ The ADCS-ADL is a 19 -item scale assessing basic and complex abilities in people with dementia. 
Items include activities such as eating, bathing, operating taps, and switching off lights. Scores range from 0 (severe impairment) to 54 (no impairment). A third outcome was safety of treatment, whereby adverse events were recorded using a structured interview.

\section{Statistical Analysis Data Preparation}

An intent-to-treat (ITT) study design was employed, where all patients who had a baseline cognitive assessment and received at least one dose of transdermal Rivastigmine were included in the study. Missing data for the ITT group was imputed using last observation carried forward (LOCF). A $t$-test was used to determine individual differences between participants included in the ITT-LOCF group and observed cases (OC).

\section{Change in Cognition}

Change on the ADAS-Cog from baseline to week 16 and to week 24 was assessed using repeated measure analysis controlling for age, gender and education.

Patients were split into three groups based on their pattern of cognitive change from baseline to week $24 ; 1$ ) improvers, rated as a 2- or more point decline on the ADAS-Cog; 2) stable, rated as a change score of 2 to -2 on the ADAS-Cog; and 3) decliners, rated as a 2 or more increase on the ADAS-Cog. The frequency of patients in each group and mean change score was calculated for each group. A second measure of cognitive change was the clinician-rated ADCS-CGIC reported at week 24. Scores were reported as frequencies.

\section{Interaction Between Treatment Response and Severity of svCVD}

To determine whether severity of svCVD affected treatment response, a repeated measure interaction model was conducted using baseline and week 24 ADAS-Cog scores and Fazekas score as the interaction factor. Interaction models were analysed separately for each treatment responder group (improvers, patients who remained stable and decliners).

\section{Differences Between Patients Who Improved, Remained Stable and Declined in Cognition}

Differences in age, education, Fazekas, baseline cognition and baseline daily functioning between patients who improved, remained stable and those that declined was assessed using analysis of variance with post hoc Tukey's test. A Chi-square test was used to determine differences in gender.

\section{Change in Activities of Daily Living}

Change on the ADL from baseline to week 16-week 24 was assessed using repeated measure analysis, controlling for age, gender and education.

\section{Adverse Events}

Adverse events were qualitatively reported using structured interviews at week 8, week 16 and week 24. Vitals, including systolic and diastolic blood pressure, heart rate and body weight, were measured at baseline, week 16 and week 24. Differences in vitals between baseline and week 16 , week 16 and week 24, and baseline and week 24 were assessed using a paired $t$-test. We imputed missing data (baseline: blood pressure [70\%], heart rate [70\%]; week 16: blood pressure [18\%)], [18\%], weight [13\%] and week 24: blood pressure [18\%], heart rate [13\%] and weight [13\%]) with the individuals average across all three time points.

\section{Availability of Data}

Data are available upon reasonable request.

\section{Results \\ Participants}

From the 100 patients recruited, there were 69 OC that had full data at all three time points. Following the ITT model, LOCF was conducted for 15 patients $(15 \%)$ at week 16 , and for 14 patients (14\%) at week 24. Reasons for withdrawal mainly included adverse events after treatment (Figure 1). No differences in cognitive or functional scores were observed between $\mathrm{OC}$ and LOCF groups at baseline (Table 1).

\section{Change in Global Cognition}

Overall, the mean change on the ADAS-COG from baseline to week 16 was $1.27(\mathrm{SD}=5.63)$. The mean change on the ADAS-Cog from baseline to week 24 was 1.78 $(\mathrm{SD}=5.29)$. Repeated measure analysis indicated that there were no significant differences on the ADAS-Cog from baseline, to week 16 to week 24 .

Figure 2 shows that $25 \%$ of the sample experienced a significant improvement in cognition after 24 weeks of treatment ( 2 or more-point decline on the ADAS-Cog). For these treatment responders, the mean change on the ADAS-Cog was $-5.08(\mathrm{SD}=3.11)$. Performance on the ADAS-Cog remained stable for $27 \%$ of the sample, with mean change $0.38(\mathrm{SD}=0$ 0.71). A decline in performance 


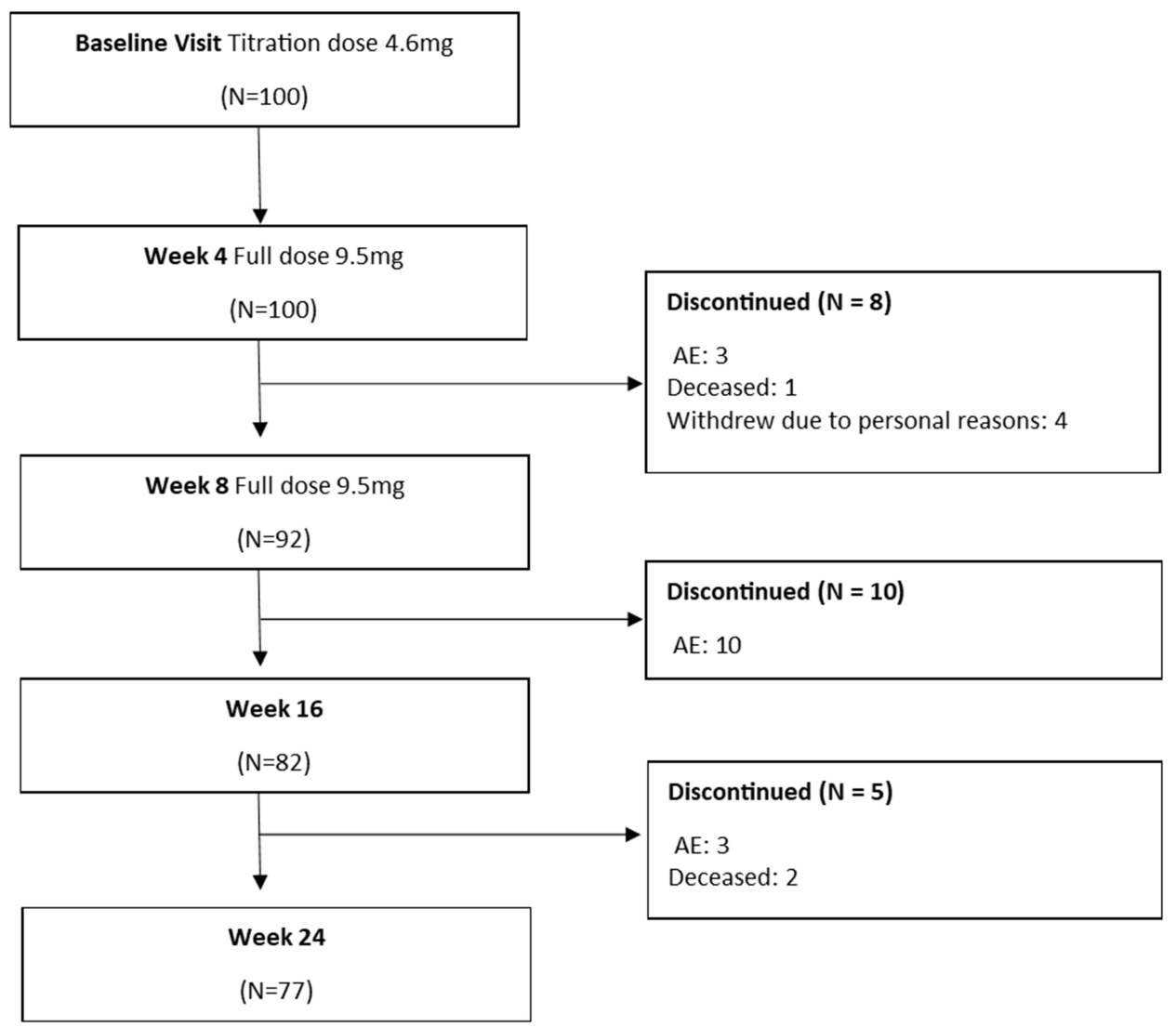

Figure I Consort diagram.

on the ADAS-Cog was observed in $49 \%$ of the sample, with a mean ADAS-Cog change of $6(\mathrm{SD}=2.98)$.

On the clinician-rated ADCS-CGIC, the mean impression of change in global function was "no change", reported for $81 \%$ of the patients (Figure 3). Minimal improvement was rated for $5 \%$ of the patients, while worsening in global function was rated for $14 \%$ of the patients.

\section{Interaction Between Treatment Response and Severity of svCVD}

Overall, there was no interaction effect between Fazekas score and treatment response on the ADAS-Cog. When looking at the three treatment groups individually, interactions remained insignificant.

\section{Differences Between Patients Who Improved, Remained Stable and Declined in Cognition}

Comparing differences in individual characteristics between patients who improved, remained stable and declined in cognition over the 24-week period, there were no differences in age, Fazekas score or cognition, as indexed using the MMSE and ADAS-Cog, or baseline daily functioning, indexed using the ADL (Table 2). Patients that remained stable had significantly more years of education compared to patients that improved or declined.

\section{Daily Functioning}

Repeated measure analysis indicated that there were no significant differences on the ADL from baseline, to week 16 to week 24, while controlling for age, gender and education.

\section{Safety}

Treatment was not associated with any serious adverse events as reported by clinical interview and analysis of vitals. The structured interview indicated that mild adverse events were observed at the 8 -week time point by $3 \%$ of the patients (Figure 1). At the 16-week time point, $10 \%$ of the patients experienced mild adverse events and at the 24-week time point, $3 \%$ of the patients experienced adverse events. The most reported adverse event was rash, limited to the site of 
Table I Participant Characteristics

\begin{tabular}{|c|c|c|c|c|c|c|}
\hline & \multicolumn{2}{|c|}{$O C(N=69)$} & \multicolumn{3}{|c|}{ ITT-LOCF (N = I00) } & \multirow[t]{2}{*}{$P$ value } \\
\hline & Mean & Std. Deviation & Mean & Std. Deviation & T-Statistic (DF) & \\
\hline Age & 74.38 & 6.98 & - & - & - & - \\
\hline Males (\%) & $48(48 \%)$ & - & - & - & - & - \\
\hline Body weight (kg) & 59 & 11.48 & & & & \\
\hline Blood pressure - Systolic & 137.45 & 19.09 & & & & \\
\hline Blood pressure - diastolic & 71.03 & 12.13 & & & & \\
\hline Heart rate (beats per min) & 67.52 & 10.25 & & & & \\
\hline Years of education & 7.58 & 4.64 & - & - & - & - \\
\hline Fazekas Score & 2.23 & 0.42 & - & - & - & - \\
\hline \multicolumn{7}{|l|}{ MMSE } \\
\hline Baseline (OC N=99) & 21.45 & 4.48 & 21.52 & 4.45 & $0.11(197)$ & 0.91 \\
\hline Week I6 (OC N=8I) & 20.12 & 4.63 & 20.51 & 4.74 & $0.55(179)$ & 0.57 \\
\hline Week 24 (OC N=87) & 20.58 & 5.21 & 20.83 & 5.04 & $0.33(185)$ & 0.73 \\
\hline \multicolumn{7}{|l|}{ ADAS Cog } \\
\hline Baseline (OC N=95) & 48.93 & 8.72 & 48.93 & 8.12 & $0.00(193)$ & 1.00 \\
\hline Week I6 (OC N=75) & 50.42 & 9.06 & 50.21 & 9.21 & $-0.15(173)$ & 0.88 \\
\hline Week $24(\mathrm{OC} \mathrm{N}=8 \mathrm{I})$ & 51.25 & 9.47 & 50.72 & 9.59 & $-.35(179)$ & 0.72 \\
\hline \multicolumn{7}{|l|}{ ADL } \\
\hline Baseline $(O C=100)$ & 61.19 & 10.26 & 61.19 & 10.26 & - & - \\
\hline Week $16(\mathrm{~N}=8 \mathrm{I})$ & 60.39 & 11.42 & 60.26 & 11.93 & $0.07(179)$ & 0.94 \\
\hline Week $24(N=85)$ & 61.02 & 10.96 & 60.43 & 11.58 & $0.35(183)$ & 0.73 \\
\hline
\end{tabular}

Abbreviations: OC, observed cases; ITT-LOCF, intent to treat-last observation carried forward sample; MMSE, Mini-Mental State Examination; ADAS-Cog, Alzheimer's disease Assessment Scale-Cognitive subscale; ADL, activities of daily living.

application of the transdermal patch (Table 3). Analysis of vitals indicated that systolic blood pressure significantly decreased from baseline to week $16(\mathrm{t}(93)=2.01, \mathrm{p}=0.04)$ (Table 4). There were no differences from week 16 to week 24 , and from baseline to week 24. A significant decrease in body weight was observed from baseline to week $24(\mathrm{t}(99)=$ $-2.24, p=0.02)$. No differences in body weight were observed between baseline and week 16, and week 16 to

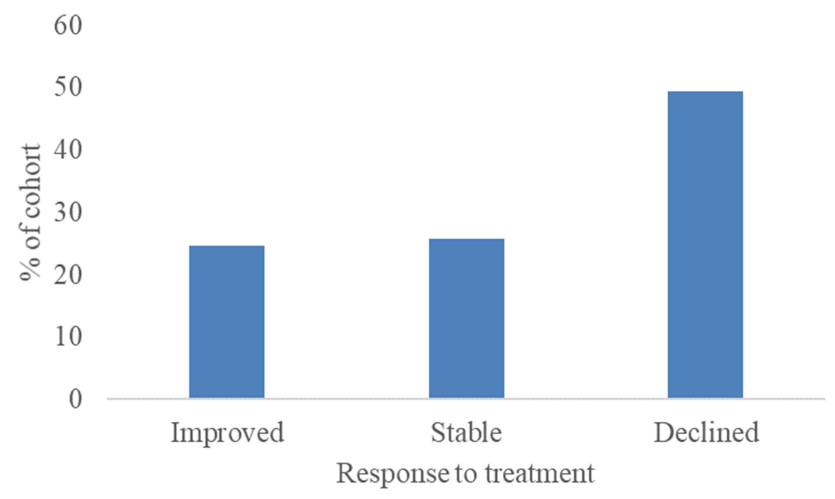

Figure 2 The proportion of patients that either responded to treatment and improved in cognitive performance, remained stable or that declined in cognitive performance after 24 weeks. week 24. No changes in diastolic blood pressure and heart rate were observed across the study duration. Three patients deceased during the study period (Figure 1). None of the deaths were considered to be treatment-related, with causes typical of an elderly AD population.

\section{Discussion}

This open-label study investigated the efficacy of transdermal Rivastigmine patch in patients with AD having concomitant MRI confirmed mild to severe svCVD over a 24-week period. Significant improvements in cognition after treatment was observed in a quarter of our sample, with an average 5-point improvement on the ADAS-Cog measure of global cognition. A quarter of the sample remained stable in their cognitive functions, while almost half experienced a decline in cognition, with an average 6-point decline. These findings were irrespective of svCVD severity. Clinician-rated impressions of global change suggested that majority (81\%) of patients appeared to remain stable in their global function after 24 weeks of treatment. Similarly, a measure of activities of daily living remained stable from baseline to week 24, suggesting Rivastigmine may maintain daily living 


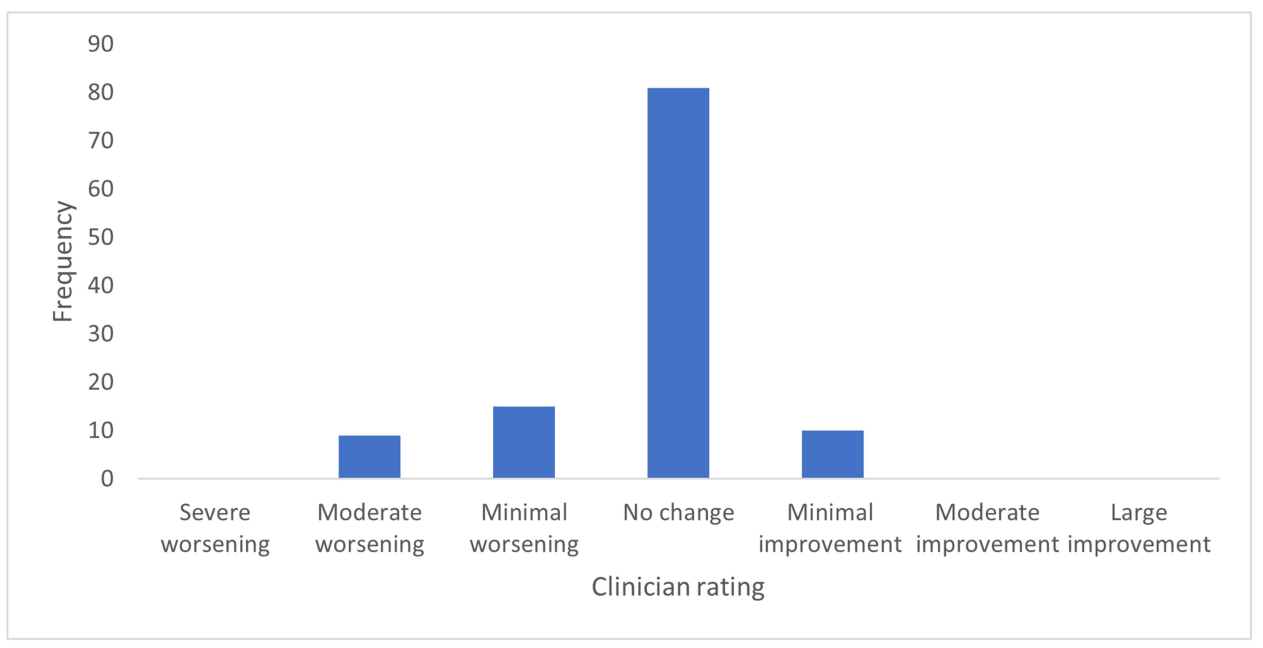

Figure 3 Frequency of responses on the clinician-rated Clinical Global Impression of Change scale at week 24.

functions. Side effects were reported by $16 \%$ of the patients, with rash being the most common. Our findings suggest that transdermal Rivastigmine may be useful in delaying cognitive decline in almost half the population of patients with $\mathrm{AD}$ having concomitant SVCVD, with minimal side effects.

Our findings are consistent with previous double-blind, placebo-controlled studies ${ }^{7}$ demonstrating that Rivastigmine slows down cognitive decline in patients with $\mathrm{AD}$ and concomitant SVCVD in a significant portion of the population. In one study, Kumar et $\mathrm{al}^{7}$ found that in a cohort of patients with $\mathrm{AD}$ and $\mathrm{sVCVD}$, indexed using the clinician-rated Modified Hachinski Ischemic Scale (MHIS), 32\% of the sample experienced a $>4$-point improvement on the ADAS-Cog. Similarly, we observed a quarter of our sample experienced a $>2$-point improvement on the ADAS-Cog. Our cohort was comparable to Kumar et $\mathrm{al}^{7}$ cohort with regards to age and

Table 2 Differences in Demographics Between Patients Who Improved, Remained Stable or Who Declined in Cognitive Performance

\begin{tabular}{|l|l|l|l|}
\hline \multirow{2}{*}{} & \multicolumn{2}{l}{ Mean (SD) } \\
\cline { 2 - 4 } & Improved & Stable & Declined \\
\cline { 2 - 4 } & $\mathbf{( N = ~ 2 5 )}$ & $\mathbf{( N = ~ 2 7 )}$ & $\mathbf{( N = ~ 4 8 )}$ \\
\hline Age & $75.61(6.27)$ & $75.04(6.44)$ & $73.26(4.09)$ \\
Gender (male) & $15(60 \%)$ & $15(55 \%)$ & $18(37 \%)$ \\
Education & $6.52(3.84)$ & $10.14(5.37)$ & $6.68(4.09)$ \\
Fazekas score & $2.32(0.47)$ & $2.25(0.44)$ & $2.16(0.37)$ \\
Baseline MMSE & $19.84(4.42)$ & $22.53(4.41)$ & $21.72(4.36)$ \\
Baseline ADAS & $48.75(9.53)$ & $51.68(8.67)$ & $47.60(8.15)$ \\
Baseline ADL & $60.36(10.35)$ & $58.40(12.55)$ & $63.18(8.42)$ \\
\hline
\end{tabular}

education, however our cohort had greater svCVD burden and baseline cognitive impairment, suggesting that the benefits of transdermal patch extend to AD patients with MRI confirmed mild-severe svCVD. Kumar et al further reported that on average, patients experienced a 1.9-point improvement on the ADAS-Cog test while the placebo group experienced a 4.2-decline in performance. In the current study, we observed that treatment responders experienced a 5-point increase in performance on the ADAS-Cog. Collectively, these findings suggest that over a quarter of the population with $\mathrm{AD}$ and concomitant SVCVD may benefit from Rivastigmine treatment, and the magnitude of improvement may be clinically relevant.

Table 3 Side Effects Reported by Clinical Interview

\begin{tabular}{|l|l|}
\hline \multicolumn{1}{|l|}{ Week 8 (N= 3) } \\
\hline & $\begin{array}{l}\text { Pruritus } \\
\text { Palpitation } \\
\text { Nausea, giddiness }\end{array}$ \\
\hline Week $16(\mathrm{~N}=10)$ \\
\hline & $\begin{array}{l}\text { Peripheral vestibulopathy } \\
\text { Neutropenia, rash } \\
\text { Rash, dyspepsia } \\
\text { Nausea, giddiness } \\
\text { Headache, behavioural change (aggression/low mood) } \\
\text { Rash contact dermatitis (N=4) }\end{array}$ \\
\hline Week 24 (N=3) \\
\hline \multicolumn{2}{|l|}{$\begin{array}{l}\text { Rash (N=2) } \\
\text { Worsening behavioral symptoms }\end{array}$} \\
\hline
\end{tabular}


Table 4 Vitals Measured at Baseline, Week 16 and Week 24

\begin{tabular}{|l|l|l|l|}
\hline Vitals & Timeframe & Mean & SD \\
\hline Systolic blood pressure (mmHg) & Baseline & 142.28 & 29.24 \\
& Week I6 & 137.76 & 19.72 \\
& Week 24 & 138.28 & 20.28 \\
\hline Diastolic blood pressure (mmHg) & Baseline & 70.74 & 10.54 \\
& Week 16 & 69.87 & 10.90 \\
& Week 24 & 70.31 & II.74 \\
\hline Heart rate (beats per min) & Baseline & 72.43 & 10.68 \\
& Week 16 & 72.20 & 12.48 \\
& Week 24 & 67.98 & 20.85 \\
\hline \multirow{2}{*}{ Weight $(\mathrm{kg})$} & Baseline & 58.76 & 11.48 \\
& Week 16 & 58.46 & 12.05 \\
& Week 24 & 58.31 & 12.01 \\
\hline
\end{tabular}

In $\mathrm{AD}$ without svCVD, the benefits of Rivastigmine is often small and some have argued that it may have little clinical importance. ${ }^{17,18}$ Previous clinical studies in patients with $\mathrm{AD}$ and no svCVD reported that transdermal patch has on average a 0.5 point improvement on the ADS- $\operatorname{Cog}^{3}$ or up to a 2 point decline in performance., ${ }^{4,19}$ In a meta-analysis with 13 placebo-controlled clinical trials in patients with $\mathrm{AD},{ }^{17}$ the mean difference between the treatment group and the placebo group on the ADASCog was 1.79 points. In comparison, Kumar et $\mathrm{al}^{7}$ reported that the treatment difference between the treatment group and placebo group for patients with $\mathrm{AD}$ having concomitant svCVD was 6.15 points, while the treatment difference for $\mathrm{AD}$ without svCVD was 4.03 points. These findings suggest that a more responsive treatment population to Rivastigmine patch may be patients with $\mathrm{AD}$ having concomitant svCVD.

One possible mechanism for the favorable effect of rivastigmine on patients with SVCVD may be its capacity to modify cardiovascular risk factors such as blood pressure. A recent study in Parkinson's disease ${ }^{20}$ demonstrated that 24 weeks of rivastigmine capsule was more effective in improving cognition compared to placebo in the hypotensive group than the non-hypotensive group. This suggests that rivastigmine may have a larger function on cognitive function by attenuating hypotension, rather than its enhancing effect on brain cholinergic tone. Thus, patients with subcortical dementias such as those with svCVD or Parkinson's disease may experience not only the acetylcholine inhibition activity of Rivastigmine but also a powerful butyryl-cholinesterase inhibition effect. ${ }^{6}$ In addition to the cognitive benefits, we observed that daily living functions remained stable over the treatment period. This finding is consistent with AD studies, whereby compared to the treatment group, placebo groups exhibited large declines in daily functioning. ${ }^{3}$ In the present study, we further observed minimal adverse events. The percentage of patients affected by adverse effects in the current study $(16 \%)$ is consistent with the safety profile of Rivastigmine reported in pure $\mathrm{AD}$ patients. ${ }^{21}$ Thus, we propose Rivastigmine patch is safe in patients with $\mathrm{AD}$ having concomitant mild to severe svCVD.

One strength of our study was that we used MRI biomarkers to confirm svCVD. All patients had moderate to severe $\mathrm{WMH}$; thus, our findings are specific to this population. Another strength is that we used a naturalistic open-label study design which allowed us to test efficacy and safety in a real-world setting. One limitation of our study was the lack of a control group. A control group of pure $\mathrm{AD}$ or pure vascular disease would have informed us whether rivastigmine is superior in treating mixed dementia with svCVD, compared to pure dementia as previously suggested. ${ }^{7,22}$ Future research may benefit from collecting AD biomarkers and MRI to discriminate between pure $\mathrm{AD}$ and $\mathrm{AD}$ with svCVD. We note we did not report cardiovascular risk factors of patients, which may have played a role in cognitive impairment in addition to the presence of WMH. ${ }^{23}$ Previous research has shown that rivastigmine is sensitive to the severity of cardiovascular risk factors ${ }^{7}$ and future research would benefit from determining whether they act as moderators to treatment effects in patients with $\mathrm{AD}$ and concomitant svCVD". We note that $29 \%$ of the data required imputation; however, we do not suspect any bias in the data as there were no group differences between patients that had imputed data vs patients with complete data.

\section{Conclusion}

We demonstrate that transdermal Rivastigmine patch is effective in targeting cognitive impairment in patients with $\mathrm{AD}$ with concomitant mild to severe svCVD, with minimal side effects. Transdermal Rivastigmine may demonstrate a huge benefit in cognition in a quarter of patients with $\mathrm{AD}$ and concomitant svCVD, with clinically relevant effects on cognitive performance. Clinical significance of these findings are important given patients with $\mathrm{AD}$ with concomitant svCVD are highly comorbid and that vascular injury is a large risk for the progression of $\mathrm{AD}$ pathology. ${ }^{24,25}$ 


\section{Trial Registration}

Health Science Authority, CTA9900218. Registered 28/11/ 2014.

\section{Data Sharing Statement}

Data will be made available upon reasonable request to corresponding author.

\section{Acknowledgments}

We would like to thank National Neuroscience Institute, Singapore, for sponsoring this study.

\section{Author Contributions}

All authors made substantial contributions to conception and design, acquisition of data, or analysis and interpretation of data; took part in drafting the article or revising it critically for important intellectual content; agreed to submit to the current journal; gave final approval of the version to be published; and agree to be accountable for all aspects of the work.

\section{Disclosure}

Novartis (Singapore) Pte Ltd partially funded the study and the cost of the investigational product. Novartis did not have any role in the concept of the study, data analyses or writing of the manuscript. This was an investigator-initiated study wherein the study design, data collection, data analyses as well as writing of this manuscript was carried out by the study team at NNI with no involvement from Novartis. The principal investigator and study team members did not receive any honorarium or personal fees from Novartis for carrying out this study. However, Dr Nagaendran Kandiah received honorarium from Novartis for lectures not related to any aspect of this study. Dr Nagaendran Kandiah reports grants from Novartis Pharmaceuticals, during the conduct of the study; and received honoraria for speaker engagements and personal fees from Novartis Pharmaceuticals, and honoraria and grant support from Eisai Pharmaceuticals and Schwabe Pharmaceuticals, outside the submitted work. The authors report no other potential conflicts of interest for this work.

\section{References}

1. Esiri MM, Joachim C, Sloan C, et al. Cerebral subcortical small vessel disease in subjects with pathologically confirmed alzheimer disease: a clinicopathologic study in the Oxford Project to Investigate Memory and Ageing (OPTIMA). Alzheimer Dis Assoc Disord. 2014;28:30-35. doi:10.1097/WAD.0b013e31829b72f1

2. White L, Petrovitch H, Hardman J, et al. Cerebrovascular pathology and dementia in autopsied Honolulu-Asia Aging Study participants. Ann N Y Acad Sci. 2002;977:9-23. doi:10.1111/j.1749-6632.2002.tb04794.x
3. Winblad B, Grossberg G, Frölich L, et al. IDEAL: a 6-month, double-blind, placebo-controlled study of the first skin patch for alzheimer disease. Neurology. 2007;69(Issue 4, Supplement 1):S14S22. doi:10.1212/01.wnl.0000281847.17519.e0

4. Feldman HH, Lane R. Rivastigmine: a placebo controlled trial of twice daily and three times daily regimens in patients with alzheimer's disease. J Neurol Neurosurg Psychiatry. 2007;78:1056-1063. doi:10.1136/jnnp.2006.099424

5. Cummings J, Froelich L, Black SE, et al. Randomized, double-blind, parallel-group, 48-week study for efficacy and safety of a higher-dose rivastigmine patch (15 vs. $10 \mathrm{~cm} 2)$ in alzheimer's disease. Dement Geriatr Cogn Disord. 2012;33:341-353. doi:10.1159/000340056

6. Kandiah N, Pai M-C, Senanarong V, et al. Rivastigmine: the advantages of dual inhibition of acetylcholinesterase and butyrylcholinesterase and its role in subcortical vascular dementia and parkinson's disease dementia. Clin Interv Aging. 2017;12:697. doi:10.2147/CIA. S129145

7. Kumar V, Anand R, Messina J, Hartman R, Veach J. An efficacy and safety analysis of exelon ${ }^{\circledR}$ in alzheimer's disease patients with concurrent vascular risk factors. Eur $J$ Neurol. 2000;7:159-169. doi:10.1046/j.1468-1331.2000.00046.x

8. Erkinjuntti T, Skoog I, Lane R, Andrews C. Rivastigmine in patients with Alzheimer's disease and concurrent hypertension. Int $J$ Clin Pract. 2002;56:791-796.

9. Debette S, Markus H. The clinical importance of white matter hyperintensities on brain magnetic resonance imaging: systematic review and meta-analysis. BMJ. 2010;341:c3666. doi:10.1136/bmj.c3666

10. McKhann GM, Knopman DS, Chertkow H, et al. The diagnosis of dementia due to alzheimer's disease: recommendations from the National Institute on Aging-Alzheimer's Association workgroups on diagnostic guidelines for alzheimer's disease. Alzheimers Dement. 2011;7(3):263-269. doi:10.1016/j.jalz.2011.03.005

11. Morris JC. The Clinical Dementia Rating (CDR): current version and scoring rules. Neurology. 1993;43(11):2412. doi:10.1212/ WNL.43.11.2412-a

12. Fazekas F, Kleinert R, Offenbacher H, et al. Pathologic correlates of incidental MRI white matter signal hyperintensities. Neurology. 1993;43:1683. doi:10.1212/WNL.43.9.1683

13. Folstein MF, Folstein SE, McHugh PR. "Mini-mental state": a practical method for grading the cognitive state of patients for the clinician. J Psychiatr Res. 1975;12:189-198. doi:10.1016/0022-3956(75)90026-6

14. Rosen W, Mohs R, Davis K. Alzheimer's Disease Assessment Scale - Cognitive and Non-Cognitive sections (ADAS-Cog, ADAS NonCog). J Psychiatry. 1984;141:1356-1364.

15. Schneider L, Olin J, Doody R, Morris JRB, Schmitt F. Validity and reliability of the ADCSU clinical global impression of change (ADCS-CGIC). Alzheimer Dis Assoc Disord. 1997;11:522-532. doi:10.1097/00002093-199700112-00004

16. Galasko D, Bennett D, Sano M, et al. An inventory to assess activities of daily living for clinical trials in alzheimer's disease. Alzheimer Dis Assoc Disord. 1997;11:33-39. doi:10.1097/00002093-199700112-00005

17. Birks JS, Evans JG. Rivastigmine for alzheimer's disease. Cochrane Database Syst Rev. 2015;4.

18. Feldman HH, Ferris S, Winblad B, et al. Effect of rivastigmine on delay to diagnosis of alzheimer's disease from mild cognitive impairment: the InDDEx study. Lancet Neurol. 2007;6(6):501-512. doi:10.1016/S1474-4422(07)70109-6

19. Nakamura Y, Imai Y, Shigeta M, et al. A 24-week, randomized, doubleblind, placebo-controlled study to evaluate the efficacy, safety and tolerability of the rivastigmine patch in Japanese patients with alzheimer's disease. Dement Geriatr Cogn Dis Extra. 2011;1:163-179. doi:10.1159/000328929

20. Espay AJ, Marsili L, Mahajan A, et al. Rivastigmine in parkinson's disease dementia with orthostatic hypotension. Ann Neurol. 2021;89 (1):91-98. doi:10.1002/ana.25923 
21. Moretti R, Torre P, Antonello RM, Cazzato G. Rivastigmine in subcortical vascular dementia: a comparison trial on efficacy and tolerability for 12 months follow-up. Eur J Neurol. 2001;8:361-362. doi:10.1046/j.1468-1331.2001.00224.x

22. Noufi P, Khoury R, Jeyakumar S, Grossberg GT. Use of cholinesterase inhibitors in non-alzheimer's dementias. Drugs Aging. 2019; $1-13$.

23. Faraco G, Iadecola C. Hypertension: a harbinger of stroke and dementia. Hypertens. 2013;62(5):810-817.
24. Zhang X, Zhou K, Wang R, et al. Hypoxia-inducible factor $1 \alpha$ (HIF$1 \alpha$ )-mediated hypoxia increases BACE1 expression and $\beta$-amyloid generation. J Biol Chem. 2007;282:10873-10880. doi:10.1074/jbc. M608856200

25. Hachinski V, Munoz DG. Cerebrovascular pathology in alzheimer's disease: cause, effect or epiphenomenon? Ann $N$ Y Acad Sci. 1997;826:1-6. doi:10.1111/j.1749-6632.1997.tb48456.x
Clinical Interventions in Aging

\section{Publish your work in this journal}

Clinical Interventions in Aging is an international, peer-reviewed journal focusing on evidence-based reports on the value or lack thereof of treatments intended to prevent or delay the onset of maladaptive correlates of aging in human beings. This journal is indexed on PubMed Central, MedLine, CAS, Scopus and the Elsevie
Bibliographic databases. The manuscript management system is completely online and includes a very quick and fair peer-review system, which is all easy to use. Visit http://www.dovepress.com/ testimonials.php to read real quotes from published authors. 